\title{
Delay Model of Hematopoietic Stem Cell Dynamics: Asymptotic Stability and Stability Switch
}

\author{
F. Crauste* \\ Université de Lyon, Université Lyon1, CNRS UMR 5208 Institut Camille Jordan \\ F - 69200 Villeurbanne Cedex, France
}

\begin{abstract}
A nonlinear system of two delay differential equations is proposed to model hematopoietic stem cell dynamics. Each equation describes the evolution of a sub-population, either proliferating or nonproliferating. The nonlinearity accounting for introduction of nonproliferating cells in the proliferating phase is assumed to depend upon the total number of cells. Existence and stability of steady states are investigated. A Lyapunov functional is built to obtain the global asymptotic stability of the trivial steady state. The study of eigenvalues of a second degree exponential polynomial characteristic equation allows to conclude to the existence of stability switches for the unique positive steady state. A numerical analysis of the role of each parameter on the appearance of stability switches completes this analysis.
\end{abstract}

Key words: nonlinear delay differential system, second degree exponential polynomial, Lyapunov function, stability switch, hematopoietic stem cell dynamics

AMS subject classification: 34K99, 34K60, 34D20, 11D61, 92C37

\section{Introduction}

In a previous publication (Crauste [11]), we considered a nonlinear model of hematopoietic stem cell dynamics, inspired by the model of Mackey [16]. It consists in a system of two delay differential equations describing the evolution of a proliferating and a nonproliferating cell population. A nonlinear term relates the dynamics of both populations.

\footnotetext{
*E-mail: crauste@math.univ-lyon1.fr
} 
Mackey's model [16] has been proposed at the end of the seventies, based on previous works by Lajtha [15] and Burns and Tannock [9], to describe the dynamics of hematopoietic stem cells and related diseases. Since then it has been improved and analyzed by many authors, including Mackey and co-authors $[17,18,20,21]$ and Adimy et al. [1, 2, 3, 4]. All these works considered that the nonlinear term, which describes the rate of introduction of nonproliferating cells in the proliferating compartment, depends only on the nonproliferating cell number. This leads to an uncoupled system of delay differential equations, in which the nonproliferating cell population dynamics prevail. It has been justified (for instance by Mackey [16]) as follows: the activation of nonproliferating cells depends on the number of free receptors at their surface. Even though this seems biologically correct and is widely accepted, it does not take the number of proliferating cells into account in the activation of nonproliferating cells. One question may arise: Is the number of activated cells independent of the number of actually proliferating cells ? Since a majority of hematopoietic stem cells $(95 \%)$ is known to be nonproliferating (see $[1,16]$ ), one may suppose that a control operates on the proportion of proliferating cells, and the total number of hematopoietic stem cells probably plays a role in the introduction of nonproliferating cells in the proliferating compartment. The nature of this role cannot be detailed yet. One may imagine very complex relations, but in this work we simply investigate the particular case when the total cell population (that is the number of proliferating and nonproliferating cells) triggers the introduction in the proliferating phase.

Such a model has already been investigated in [11], in a particular case when the mortality rates of proliferating and nonproliferating cells were assumed to be equal. This situation is very restrictive, and not biologically reasonable. Nevertheless, the mathematical analysis of the model proved to be clear and easier to present. In this paper, we consider the general case, with no assumption on the mortality rates, except that they are nonnegative quantities. The delay system we obtain is not decoupled and, in particular, the analysis of the local asymptotic stability is performed through the study of eigenvalues of a second degree exponential polynomial characteristic equation with delay-dependent coefficients.

Before giving a brief summary of the work presented in this paper, let us recall the basis of hematopoietic stem cell dynamics. Hematopoiesis, the blood production process, is based on a cell population divided in two compartments, a proliferating and a nonproliferating phase, able to produce, by successive divisions, all types of blood cells (white cells, red blood cells and platelets). Nonproliferating cells are in a quiescent stage, where they can stay their entire life. They are able to differentiate in more mature cells, and they are introduced in the proliferating phase with a nonlinear rate (as described above). Proliferating cells can die by apoptosis, a programmed cell death, and divide after a given time. They produce two daughter cells that immediately enter the nonproliferating phase. A destabilization of this system can cause hematological diseases, such as leukemias or anemias [14]. Some of these blood diseases are characterized by temporal oscillations of blood cell counts [12], and then a mathematical analysis of hematopoietic stem cell dynamics can be a useful tool to determine the causes of the destabilization. For instance, Pujo-Menjouet and Mackey [21] and Pujo-Menjouet et al. [20] gave an insight into Mackey's model [16] by highlighting the role of each parameter of the model on the appearance of periodic solutions, and applied the model to the study of chronic myelogenous leukemia [12]. Bernard et al. [8] used a similar approach to investigate the causes of cyclical neutropenia, an other periodic hematological 
disease.

The model we focus on in this paper consists in two nonlinear delay differential equations, where the delay accounts for cell cycle duration and the nonlinear term depends on the sum of the two variables (see (2.3)-(2.4)). We investigate the existence of steady states, and their stability. The global asymptotic stability of the trivial steady state, describing cell's dying out, is obtained by constructing a Lyapunov functional in Section 4. Then, we concentrate ourselves in Section 5 on the local stability of the unique positive steady state. The linearization of the initial system leads to a second degree exponential polynomial with delay-dependent coefficients. Such equations are known to create stability switches $[2,7,11]$. We analyze the existence of stability switches for the positive steady state. This study is completed by a numerical investigation of the role of each parameter on the stability of the system in Section 6, which completes the analysis. We conclude with a discussion, showing in particular that the model we propose exhibits similarities with Mackey's model [16] and gives interesting prospects regarding the behavior of hematopoietic cell populations.

\section{A delay system of blood cell production}

We consider the classical Mackey's model [16],

$$
\begin{aligned}
& \frac{d N}{d t}(t)=-(\delta+\beta) N(t)+2 e^{-\gamma \tau} \beta N(t-\tau), \\
& \frac{d P}{d t}(t)=-\gamma P(t)+\beta N(t)-e^{-\gamma \tau} \beta N(t-\tau),
\end{aligned}
$$

describing the evolution of a hematopoietic stem cell population, located in the bone marrow. The population consists in two distinct sub-populations, nonproliferating and proliferating cells, whose densities are respectively denoted by $N(t)$ and $P(t)$.

Nonproliferating cells differentiate with a constant rate $\delta \geq 0$, and are introduced in the proliferating phase with a nonnegative rate $\beta$. Proliferating cells die by apoptosis (programmed cell death) with a rate $\gamma \geq 0$, and divide after a time $\tau$. The last term in (2.1) accounts for proliferating cells that have performed a complete cell cycle, divided, and then enter a new nonproliferating stage. These cells are in fact nonproliferating cells introduced in the proliferating phase a time $\tau$ earlier. This creates delayed terms in system (2.1)-(2.2).

Similarly to what we did in [11], we assume the rate of introduction $\beta$ depends on the total population of hematopoietic stem cells, denoted by $S$. Indeed, $S=P+N$. This assumption stresses the fact that the nature of the trigger signal for introduction in the proliferating phase is the result of an action on the entire cell population. For example, it can be caused by molecules entering the bone marrow and fixing on hematopoietic stem cells, activating or inhibiting their proliferating capacity. This occurs in particular for the production of red blood cells. Their regulation is mainly mediated by a hormone (a growth factor, in fact) called erythropoietin, produced by the kidneys under a stimulation by circulating blood cells (see Bélair et al. [6], Mahaffy et al. [19]). 
From now on, we assume then

$$
\beta=\beta(S(t)) .
$$

The function $\beta$ is supposed to be continuous and positive on $[0,+\infty)$, and strictly decreasing with

$$
\lim _{S \rightarrow \infty} \beta(S)=0,
$$

to take into account larger introduction of cells when there is a lack of hematopoietic stem cells (see Crauste [11], Mackey [16], Pujo-Menjouet and Mackey [21], and the references therein).

Adding equations (2.1) and (2.2), we deduce a system of delay differential equations, satisfied by $N(t)$ and $S(t)$, given by

$$
\begin{aligned}
& \frac{d N}{d t}(t)=-\delta N(t)-\beta(S(t)) N(t)+2 e^{-\gamma \tau} \beta(S(t-\tau)) N(t-\tau), \\
& \frac{d S}{d t}(t)=-\gamma S(t)+(\gamma-\delta) N(t)+e^{-\gamma \tau} \beta(S(t-\tau)) N(t-\tau) .
\end{aligned}
$$

For a given initial condition $(\varphi, \psi)$, defined on the interval $[-\tau, 0]$, system (2.3)-(2.4) has a unique continuous solution $\left(N^{\varphi}(t), S^{\psi}(t)\right.$ ), well-defined for $t \geq 0$ (see Hale and Verduyn Lunel [13]). Moreover, solutions are positive, as stated in the following lemma.

Lemma 1. For all nonnegative initial conditions, the solution $N(t)$ of $(2.3)$ is nonnegative. Moreover, assuming

$$
S(0) \geq N(0)+\int_{-\tau}^{0} e^{\gamma \theta} \beta(S(\theta)) N(\theta) d \theta,
$$

the solution $(N(t), S(t))$ satisfies, for all $t \geq 0, S(t) \geq N(t) \geq 0$.

Proof. First concentrate on the positivity of $N(t)$. Suppose there exists $T>0$ such that $N(T)=0$ and $N(t) \geq 0$ for $t<T$, and there exists $0<\varepsilon<\tau$ such that $N(t)<0$ for $t \in(T, T+\varepsilon)$. Then, from (2.3) and since $\beta$ is a positive function,

$$
\frac{d N}{d t}(t)>0 \quad \text { for all } t \in(T, T+\varepsilon)
$$

This leads to a contradiction, and consequently $N(t) \geq 0$ for all $t>0$.

Now, assume (2.5) holds true. We focus on the difference $S(t)-N(t)$, that equals $P(t)$ and satisfies equation (2.2). Hence, one easily obtains, for $t \geq 0$,

$$
e^{\gamma t}[S(t)-N(t)]-\int_{t-\tau}^{t} e^{\gamma \theta} \beta(S(\theta)) N(\theta) d \theta=S(0)-N(0)-\int_{-\tau}^{0} e^{\gamma \theta} \beta(S(\theta)) N(\theta) d \theta .
$$

From (2.5), one deduces

$$
S(t) \geq N(t)+e^{-\gamma t} \int_{t-\tau}^{t} e^{\gamma \theta} \beta(S(\theta)) N(\theta) d \theta, \quad \text { for all } t \geq 0 .
$$


Since $N$ is nonnegative and $\beta$ is positive, then

$$
\int_{t-\tau}^{t} e^{\gamma \theta} \beta(S(\theta)) N(\theta) d \theta>0 \quad \text { for all } t \geq 0
$$

and we deduce that $S(t) \geq N(t) \geq 0$ for all $t \geq 0$.

Condition (2.5) has a strong biological meaning. The integral term $\int_{-\tau}^{0} e^{\gamma \theta} \beta(S(\theta)) N(\theta) d \theta$ represents the population of cells that have been introduced in the proliferating phase at time $\theta \in$ $[-\tau, 0]$ and that have survived at time $t=0$. From a biological point of view, the population in the proliferating phase at time $t=0$ should be larger than this quantity, and this is what (2.5) indicates.

One can note that the positivity of $S(t)$ can be handled similarly to the positivity of $N(t)$ when $\gamma \geq \delta$. However, when $\gamma<\delta,(2.5)$ is necessary to obtain the positivity of $S(t)$.

Next, we investigate the existence of steady states of system (2.3)-(2.4). A steady state is a solution $(\bar{N}, \bar{S})$ satisfying

$$
\frac{d \bar{N}}{d t}=\frac{d \bar{S}}{d t}=0
$$

We deduce

$$
\begin{aligned}
{\left[\delta-\left(2 e^{-\gamma \tau}-1\right) \beta(\bar{S})\right] \bar{N} } & =0 \\
{\left[\gamma-\delta+e^{-\gamma \tau} \beta(\bar{S})\right] \bar{N} } & =\gamma \bar{S} .
\end{aligned}
$$

The following proposition states the existence of two steady states.

Proposition 2. Assume

$$
\left(2 e^{-\gamma \tau}-1\right) \beta(0)>\delta .
$$

Then system (2.3)-(2.4) has two steady states, $(\bar{N}, \bar{S})=(0,0)$ and $(\bar{N}, \bar{S})=\left(N^{*}, S^{*}\right)$, where $S^{*}>0$ is the unique solution of

$$
\left(2 e^{-\gamma \tau}-1\right) \beta\left(S^{*}\right)=\delta
$$

and

$$
N^{*}=\frac{\left(2 e^{-\gamma \tau}-1\right) \gamma}{\left(2 e^{-\gamma \tau}-1\right) \gamma+\left(1-e^{-\gamma \tau}\right) \delta} S^{*}
$$

If (2.8) does not hold, then system (2.3)-(2.4) has only one steady state, $(\bar{N}, \bar{S})=(0,0)$.

Proof. First, one notices that $(\bar{N}, \bar{S})=(0,0)$ is always a solution of (2.6)-(2.7). Moreover, one can easily check, from (2.6)-(2.7), that $\bar{N}=0$ if and only if $\bar{S}=0$.

Let search for solutions of (2.6)-(2.7) such that $\bar{N}>0$ and $\bar{S}>0$. From (2.6),

$$
\left(2 e^{-\gamma \tau}-1\right) \beta(\bar{S})=\delta
$$

and, from (2.7),

$$
\bar{N}=\frac{\gamma \bar{S}}{\gamma-\delta+e^{-\gamma \tau} \beta(\bar{S})} .
$$


Since $\beta$ is decreasing and tends to zero at infinity, equation (2.9) has a solution, which is unique, if and only if (2.8) holds true. Using (2.9) in the expression for $\bar{N}$ above, one obtains the expression of $N^{*}$. This ends the proof.

Condition (2.8) is equivalent to

$$
\delta<\beta(0) \quad \text { and } \quad 0 \leq \tau<\tau_{\max }:=\frac{1}{\gamma} \ln \left(\frac{2 \beta(0)}{\delta+\beta(0)}\right)
$$

Thus, the positive steady state exists provided that the maximum rate of introduction is larger than the mortality rate $\delta$, and the cell cycle duration $\tau$ is not too large. One can note, in particular, that when the unique positive steady state exists $S^{*} \geq N^{*}$ and $2 e^{-\gamma \tau}-1>0$.

The next section is devoted to the linearization of system (2.3)-(2.4) about one of its steady states to analyze its local asymptotic stability.

\section{Linearization and characteristic equation}

The linearization of system (2.3)-(2.4) will allow analyzing the asymptotic behavior of its solutions. We assume $\beta$ is continuously differentiable on $[0,+\infty)$.

Let $(\bar{N}, \bar{S})$ be a steady state of system (2.3)-(2.4), and define

$$
\bar{\alpha}=\bar{\alpha}(\bar{N}, \bar{S}):=\bar{N} \beta^{\prime}(\bar{S}) \quad \text { and } \quad \bar{\beta}:=\beta(\bar{S}) .
$$

System (2.3)-(2.4) linearized about $(\bar{S}, \bar{N})$ writes

$$
\left(\begin{array}{l}
\frac{d N}{d t}(t) \\
\frac{d S}{d t}(t)
\end{array}\right)=\mathcal{A}_{1}\left(\begin{array}{c}
N(t) \\
S(t)
\end{array}\right)+\mathcal{A}_{2}\left(\begin{array}{c}
N(t-\tau) \\
S(t-\tau)
\end{array}\right)
$$

with

$$
\mathcal{A}_{1}:=\left(\begin{array}{cc}
-(\delta+\bar{\beta}) & -\bar{\alpha} \\
\gamma-\delta & -\gamma
\end{array}\right), \quad \mathcal{A}_{2}:=e^{-\gamma \tau}\left(\begin{array}{cc}
2 \bar{\beta} & 2 \bar{\alpha} \\
\bar{\beta} & \bar{\alpha}
\end{array}\right) .
$$

The characteristic equation of the above system, associated with $(\bar{N}, \bar{S})$, is

$$
\operatorname{det}\left(\lambda-\mathcal{A}_{1}-e^{-\lambda \tau} \mathcal{A}_{2}\right)=0,
$$

that reduces to the second order exponential polynomial

$$
\Delta(\lambda, \tau):=Q_{2}(\lambda, \tau)+Q_{1}(\lambda, \tau) e^{-\lambda \tau}=0,
$$

where

$$
Q_{2}(\lambda, \tau):=\lambda^{2}+a_{1}(\tau) \lambda+a_{2}(\tau) \quad \text { and } \quad Q_{1}(\lambda, \tau):=a_{3}(\tau) \lambda+a_{4}(\tau)
$$


with

$$
\begin{array}{ll}
a_{1}(\tau)=\delta+\gamma+\bar{\beta}, & a_{2}(\tau)=\gamma(\delta+\bar{\beta})+\bar{\alpha}(\gamma-\delta), \\
a_{3}(\tau)=-(\bar{\alpha}+2 \bar{\beta}) e^{-\gamma \tau}, & a_{4}(\tau)=[\bar{\alpha}(\delta-2 \gamma)-2 \gamma \bar{\beta}] e^{-\gamma \tau} .
\end{array}
$$

One may note that coefficients $a_{i}, i=1, \ldots, 4$, depend explicitly but also implicitly on $\tau$ through the values of the steady states $\bar{N}$ and $\bar{S}$.

Let us recall that the steady state $(\bar{N}, \bar{S})$ is locally asymptotically stable when all roots of (3.2) have negative real parts, and the stability can only be lost if eigenvalues cross the imaginary axis, that is if pure imaginary roots appear.

We focus, in the next section, on the global stability of the trivial steady state. Then, in Section 5 , we will concentrate ourselves on the behavior of the unique positive steady state.

\section{Global asymptotic stability}

This section is devoted to the asymptotic behavior analysis of the trivial steady state of system (2.3)-(2.4), that is $(\bar{N}, \bar{S})=(0,0)$. First, one can note that, from (3.1),

$$
\bar{\alpha}(0,0)=0 \quad \text { and } \quad \bar{\beta}(0)=\beta(0),
$$

so the characteristic equation (3.2) reduces to

$$
[\lambda+\gamma]\left[\lambda+\delta+\beta(0)-2 \beta(0) e^{-\gamma \tau} e^{-\lambda \tau}\right]=0 .
$$

Throughout this section, we assume (2.5) holds true, so solutions of (2.3)-(2.4) are nonnegative.

Before stating a necessary and sufficient condition for the asymptotic stability of the trivial steady state, we prove the next lemma.

Lemma 3. Let $(N(t), S(t))$ be a bounded solution of (2.3)-(2.4). Then $\lim _{t \rightarrow+\infty} N(t)=0$ if and only if $\lim _{t \rightarrow+\infty} S(t)=0$.

Proof. We first prove that $\lim _{t \rightarrow+\infty} N(t)=0$ implies $\lim _{t \rightarrow+\infty} S(t)=0$. From (2.4), and using a classical variation of constant formula, then, for $t \geq 0, S(t)$ is given by

$$
S(t)=e^{-\gamma t} S(0)+(\gamma-\delta) e^{-\gamma t} \int_{0}^{t} N(\theta) e^{\gamma \theta} d \theta+e^{-\gamma t} \int_{-\tau}^{t-\tau} e^{\gamma \theta} \beta(S(\theta)) N(\theta) d \theta .
$$

Let $\varepsilon>0$ be fixed. Since $N$ tends to zero when $t$ tends to $\infty$, there exists $T>0$ such that

$$
N(t)<\widetilde{\varepsilon}:=\frac{\varepsilon}{2} \frac{\gamma}{|\gamma-\delta|+e^{-\gamma \tau} \beta(0)}, \quad \text { for } t \geq T \text {. }
$$

Then, setting

$$
C(T):=S(0)+(\gamma-\delta) \int_{0}^{T} N(\theta) e^{\gamma \theta} d \theta+\int_{-\tau}^{T} e^{\gamma \theta} \beta(S(\theta)) N(\theta) d \theta,
$$


one obtains, for $t \geq T+\tau$, using (4.2) and the fact that $\beta(0)$ is a bound of $\beta$,

$$
\begin{aligned}
|S(t)| & \leq e^{-\gamma t}|C(T)|+|\gamma-\delta| \widetilde{\varepsilon} e^{-\gamma t} \int_{T}^{t} e^{\gamma \theta} d \theta+\beta(0) \widetilde{\varepsilon} e^{-\gamma t} \int_{T}^{t-\tau} e^{\gamma \theta} d \theta \\
& \leq e^{-\gamma t}|C(T)|+\frac{\widetilde{\varepsilon}}{\gamma}\left[|\gamma-\delta|\left(1-e^{-\gamma(t-T)}\right)+\beta(0) e^{-\gamma \tau}\left(1-e^{-\gamma(t-T-\tau)}\right)\right] \\
& \leq e^{-\gamma t}|C(T)|+\frac{\varepsilon}{2}
\end{aligned}
$$

Let $\bar{t}>0$ be such that

$$
e^{-\gamma t}|C(T)|<\frac{\varepsilon}{2}, \quad \text { for } t \geq \bar{t}
$$

Then, for $t \geq \max \{\bar{t}, T+\tau\}$, one obtains

$$
|S(t)|<\varepsilon
$$

Hence $\lim _{t \rightarrow+\infty} S(t)=0$.

Conversely, assume $\lim _{t \rightarrow+\infty} S(t)=0$. Since, from Lemma $1, S(t) \geq N(t) \geq 0$ for all $t \geq 0$, we deduce that $\lim _{t \rightarrow+\infty} N(t)=0$. This ends the proof.

We are then able to state and prove the following theorem, dealing with the global asymptotic stability of the trivial steady state.

Theorem 4. All solutions $(N(t), S(t))$ of (2.3)-(2.4) converge to the trivial steady state $(0,0)$ if and only if

$$
\left(2 e^{-\gamma \tau}-1\right) \beta(0) \leq \delta
$$

Proof. Let us set $C:=\mathcal{C}^{0}\left([-\tau, 0], \mathbb{R}^{+}\right)$, the space of continuous and nonnegative functions on $[-\tau, 0]$. We then define, for $(\varphi, \psi) \in C^{2}$,

$$
V(\varphi, \psi):=\varphi(0)+2 e^{-\gamma \tau} \int_{-\tau}^{0} \beta(\psi(\theta)) \varphi(\theta) d \theta .
$$

Using (2.3)-(2.4), one can easily check that, for $(\varphi, \psi) \in C^{2}$,

$$
\dot{V}(\varphi, \psi)=\varphi(0)\left[\left(2 e^{-\gamma \tau}-1\right) \beta(\psi(0))-\delta\right],
$$

where $\dot{V}$ is the derivative of $V$ along the solutions of (2.3)-(2.4).

Following the notations in Hale and Verduyn Lunel [13], we deduce that $V$ is a Lyapunov functional on the set

$$
G:=\left\{(\varphi, \psi) \in C^{2} ; \varphi(0)\left[\left(2 e^{-\gamma \tau}-1\right) \beta(\psi(0))-\delta\right] \leq 0\right\} .
$$

From (4.3) and since $\beta$ is a decreasing function, we obtain $G=C^{2}$. Now, let

$$
K:=\{(\varphi, \psi) \in \bar{G} ; \dot{V}(\varphi, \psi)=0\} .
$$


We first assume

$$
\left(2 e^{-\gamma \tau}-1\right) \beta(0)<\delta
$$

From (4.5) we deduce that

$$
K=\left\{(\varphi, \psi) \in C^{2} ; \varphi(0)=0\right\} .
$$

We now consider the set $M$, defined as the largest set in $K$ which is invariant with respect to (2.3)(2.4). Let $\left(N_{t}^{\varphi}, S_{t}^{\psi}\right)$ be a solution of (2.3)-(2.4) associated with the initial condition $(\varphi, \psi)$. We recall that, for all $t \geq 0$, the function $N_{t}^{\varphi}$ (respectively $S_{t}^{\psi}$ ), defined by $N_{t}^{\varphi}(\theta)=N^{\varphi}(t+\theta)$ for $\theta \in[-\tau, 0]$, belongs to $C$. Then $\left(N_{t}^{\varphi}, S_{t}^{\psi}\right) \in M$ for all $t \geq 0$ is equivalent to

$$
N^{\varphi}(t)=0 \quad \text { for } t \geq 0 .
$$

Consequently,

$$
M=\{0\} \times C .
$$

From Hale and Verduyn Lunel [13], all bounded solutions $\left(N_{t}^{\varphi}, S_{t}^{\psi}\right)$ of (2.3)-(2.4) converge to $M$ as $t$ tends to $+\infty$. Consequently, provided that all solutions of (2.3)-(2.4)) are bounded, they converge to $M$ as $t$ tends to $+\infty$, that is, for all $\varphi \in C$,

$$
\lim _{t \rightarrow+\infty} N^{\varphi}(t)=0
$$

From Lemma 3, we deduce that $\lim _{t \rightarrow+\infty} S^{\psi}(t)=0$ so $\left(N^{\varphi}(t), S^{\psi}(t)\right)$ converge to $(0,0)$.

Assume now

$$
\left(2 e^{-\gamma \tau}-1\right) \beta(0)=\delta .
$$

Then, following the notations above, we obtain

$$
\begin{aligned}
K & =\left\{(\varphi, \psi) \in C^{2} ; \varphi(0)=0 \text { or } \beta(\psi(0))=\beta(0)\right\} \\
& =\left\{(\varphi, \psi) \in C^{2} ; \varphi(0)=0 \text { or } \psi(0)=0\right\} .
\end{aligned}
$$

Using the same reasoning than above, we obtain

$$
M=(\{0\} \times C) \cup(C \times\{0\}) .
$$

Using similar arguments, we deduce that all solutions $\left(N^{\varphi}(t), S^{\psi}(t)\right)$ of (2.3)-(2.4)) converge to $(0,0)$, provided that they are bounded.

Now, let us show that nonnegative solutions of (2.3)-(2.4)) are bounded provided that (4.3) holds true. Let $(N(t), S(t))$ be a solution of (2.3)-(2.4), and for $t \geq 0$ set

$$
W(t)=V\left(N_{t}, S_{t}\right)
$$

where $V$ is defined by (4.4). Then, for $t \geq 0$,

$$
W^{\prime}(t)=N(t)\left[\left(2 e^{-\gamma \tau}-1\right) \beta(S(t))-\delta\right] \leq 0 .
$$


Consequently, $W(t)$ is bounded above, and so is $N(t)$. Using a reasoning similar to the one in the proof of Lemma 3, we deduce that $S(t)$ is bounded when $N(t)$ is bounded.

Eventually, we have proved that all solutions of system (2.3)-(2.4) converge to the trivial steady state provided that (4.3) holds true.

Let now assume (4.3) is not satisfied, that is (2.8) holds true. Eigenvalues of (4.1) are $\lambda=$ $-\gamma<0$ and roots of

$$
\Delta_{0}(\lambda):=\lambda+\delta+\beta(0)-2 \beta(0) e^{-\gamma \tau} e^{-\lambda \tau} .
$$

Consider $\Delta_{0}$ as a function of real $\lambda$. Then $\Delta_{0}$ is increasing and tends to $\infty$ as $\lambda$ tends to $\infty$. Moreover, $\Delta_{0}(0)=\delta-\left(2 e^{-\gamma \tau}-1\right) \beta(0)<0$ from (2.8). Consequently, $\Delta_{0}$ has a unique positive real root, which is an eigenvalue of (4.1). This implies the instability of the trivial steady state when (4.3) is not satisfied, and it concludes the proof.

Condition (4.3) indicates that the entire cell population dies out when mortality rates are large compared to the introduction rate. In this case, the cell population cannot persist, and asymptotically disappears.

In the next section, we study the asymptotic behavior of the unique positive steady state of (2.3)-(2.4).

\section{Stability switch for the positive steady state}

We now investigate the stability of the unique positive steady state of system $(2.3)-(2.4),\left(N^{*}, S^{*}\right)$ defined by Proposition 2. We assume (2.8) holds true — or equivalently (2.10) — throughout this section, to ensure the existence of the steady state. Moreover, we set

$$
\alpha^{*}:=\bar{\alpha}\left(N^{*}, S^{*}\right)=N^{*} \beta^{\prime}\left(S^{*}\right) \quad \text { and } \quad \beta^{*}:=\beta\left(S^{*}\right) .
$$

To determine whether the positive steady state is locally asymptotically stable or unstable, we have to study the roots of (3.2). If all roots have negative real parts, then the positive steady state is asymptotically stable, whereas it is unstable if roots with positive real parts exist.

The mapping $\Delta$, defined in (3.2), is a second degree exponential polynomial, with delaydependent coefficients. This particular form makes the study of characteristic roots rather difficult. In particular, there is no hope to analytically determine some eigenvalues.

Such equations have been studied by Beretta and Kuang [7]. They developed a theory that allows to determine stability switches (if they exist) or stability areas by simply computing the real roots of a particular function, which reveals easier to analyze than the full characteristic equation. This method has been used, for instance, by Adimy et al. [5] to study a characteristic equation similar to (3.2). In the following, we use the approach of Beretta and Kuang [7] to solve our problem. At first, we focus on the stability in the absence of delay, that is when $\tau=0$.

Proposition 5. When $\tau=0$, the positive steady state of system (2.3)-(2.4) is locally asymptotically stable. 
Proof. When $\tau=0$, the positive steady state satisfies $\beta^{*}=\delta$ and (3.2) becomes

$$
\lambda^{2}+\left[a_{1}(0)+a_{3}(0)\right] \lambda+\left[a_{2}(0)+a_{4}(0)\right]=0 .
$$

From Routh-Hurwitz criterion, all characteristic roots have negative real parts if and only if $a_{1}(0)+$ $a_{3}(0)>0$ and $a_{2}(0)+a_{4}(0)>0$.

From (3.4), we obtain

$$
a_{1}(0)+a_{3}(0)=\delta+\gamma+\beta^{*}-\alpha^{*}-2 \beta^{*}=\gamma-\alpha^{*}>0
$$

since $\alpha^{*}<0$, and

$$
a_{2}(0)+a_{4}(0)=\gamma\left(\delta+\beta^{*}\right)+\alpha^{*}(\gamma-\delta)+\alpha^{*}(\delta-2 \gamma)-2 \gamma \beta^{*}=-\gamma \alpha^{*}>0 .
$$

This concludes the proof.

Since the positive steady state is asymptotically stable when $\tau=0$, we search for values of $\tau \in\left(0, \tau_{\max }\right)$ modifying the stability of the steady state. If instability occurs for a particular $\tau_{c}$, a root of (3.2) must intersect the imaginary axis. We then investigate the existence of purely imaginary roots of (3.2), and in order to apply the theory of Beretta and Kuang [7], we first check some properties of the polynomial functions $Q_{2}$ and $Q_{1}$.

Let $\tau \in\left[0, \tau_{\max }\right)$ be given. First, one can see that

$$
Q_{2}(0, \tau)+Q_{1}(0, \tau)=a_{2}(\tau)+a_{4}(\tau)=\left[\left(e^{-\gamma \tau}-1\right) \delta+\left(1-2 e^{-\gamma \tau}\right) \gamma\right] \alpha^{*}>0
$$

Secondly,

$$
Q_{2}(i \omega, \tau)+Q_{1}(i \omega, \tau)=-\omega^{2}+a_{2}(\tau)+a_{4}(\tau)+i \omega\left[a_{1}(\tau)+a_{3}(\tau)\right] \neq 0,
$$

since, for instance,

$$
a_{1}(\tau)+a_{3}(\tau)=\gamma-e^{-\gamma \tau} \alpha^{*}>0 .
$$

Thirdly, from the definition of $Q_{2}$ and $Q_{1}$ in (3.3), it is straightforward that

$$
\limsup \left\{\left|\frac{Q_{1}(\lambda, \tau)}{Q_{2}(\lambda, \tau)}\right| ;|\lambda| \rightarrow \infty, \operatorname{Re}(\lambda) \geq 0\right\}<1
$$

Finally, let us define the polynomial function

$$
F(\omega, \tau):=\left|Q_{2}(i \omega, \tau)\right|^{2}-\left|Q_{1}(i \omega, \tau)\right|^{2} .
$$

Then

$$
F(\omega, \tau)=\omega^{4}+b_{1}(\tau) \omega^{2}+b_{2}(\tau)
$$

where

$$
b_{1}(\tau):=a_{1}^{2}(\tau)-2 a_{2}(\tau)-a_{3}^{2}(\tau) \quad \text { and } \quad b_{2}(\tau):=a_{2}^{2}(\tau)-a_{4}^{2}(\tau)
$$


It is clear that $F$ has a finite number of roots. Moreover, $F(\omega, \tau)=f\left(\omega^{2}, \tau\right)$, where $f(z, \tau):=$ $z^{2}+b_{1}(\tau) z+b_{2}(\tau)$.

Now, let search for purely imaginary roots $i \omega$ of (3.2). Then, separating real and imaginary parts in (3.2) leads to

$$
\left\{\begin{aligned}
a_{4}(\tau) \cos (\omega \tau)+a_{3}(\tau) \omega \sin (\omega \tau) & =\omega^{2}-a_{2}(\tau), \\
a_{3}(\tau) \omega \cos (\omega \tau)-a_{4}(\tau) \sin (\omega \tau) & =-a_{1}(\tau) \omega .
\end{aligned}\right.
$$

One can easily check that $\omega \neq 0$, since $a_{2}(\tau)+a_{4}(\tau)>0$ from (5.1). Moreover, if $(\omega, \tau)$ satisfies (5.2), then so does $(-\omega, \tau)$. Therefore, we only look for positive $\omega$ satisfying (5.2).

Adding the squares of both sides of equations in (5.2), one observes that $\omega$ must satisfy $F(\omega, \tau)=0$. Assume

$$
b_{2}(\tau)<0 \quad \text { or } \quad \frac{b_{1}^{2}(\tau)}{4} \geq b_{2}(\tau) \geq 0>b_{1}(\tau)
$$

Then $f(\cdot, \tau)$ has at least one positive root, therefore there exists at least one $\omega=\omega(\tau)>0$ such that $F(\omega(\tau), \tau)=0$.

Let's check that (5.3) can be satisfied for some $\tau \in\left(0, \tau_{\max }\right)$. Indeed, we focus on the first condition in (5.3). Notice that

$$
b_{2}(\tau)=a_{2}^{2}(\tau)-a_{4}^{2}(\tau)=\left[a_{2}(\tau)-a_{4}(\tau)\right]\left[a_{2}(\tau)+a_{4}(\tau)\right]
$$

and, from (5.1), $a_{2}(\tau)+a_{4}(\tau)>0$ for $\tau \in\left[0, \tau_{\max }\right)$. Consequently, $b_{2}(\tau)<0$ if and only if $a_{2}(\tau)-a_{4}(\tau)<0$. For all $\tau \in\left[0, \tau_{\max }\right)$,

$$
a_{2}(\tau)-a_{4}(\tau)=\gamma\left[\delta+\left(2 e^{-\gamma \tau}+1\right) \beta^{*}\right]+\left[\left(2 e^{-\gamma \tau}+1\right) \gamma-2 \delta\right] \alpha^{*} .
$$

Using (2.9), $\alpha^{*}=N^{*} \beta^{\prime}\left(S^{*}\right)$ and the definition of $N^{*}$ given in Proposition 2, we deduce that $a_{2}(\tau)-a_{4}(\tau)<0$ if and only if

$$
S^{*}(\tau) \beta^{\prime}\left(S^{*}(\tau)\right)<-\frac{4 \delta e^{-\gamma \tau}}{\left(2 e^{-\gamma \tau}-1\right)^{2}} \times \frac{e^{-\gamma \tau}(2 \gamma-\delta)-(\gamma-\delta)}{e^{-\gamma \tau}(2 \gamma-\delta)+\gamma-\delta}:=\xi(\tau) .
$$

Consequently, if $S^{*}(0) \beta^{\prime}\left(S^{*}(0)\right)<\xi(0)$, then $a_{2}(\tau)-a_{4}(\tau)<0$ in a neighborhood of $\tau=0$. The following proposition states this sufficient condition.

Proposition 6. Assume

$$
\beta^{-1}(\delta) \beta^{\prime}\left(\beta^{-1}(\delta)\right)<-\frac{4 \delta \gamma}{3 \gamma-2 \delta} .
$$

Then there exists $0<\bar{\tau}<\tau_{\max }$ such that $a_{2}(\tau)-a_{4}(\tau)<0$ for $\tau \in[0, \bar{\tau})$. 
Suppose now there exists $0<\bar{\tau}<\tau_{\max }$ such that (5.3) holds true for $\tau \in[0, \bar{\tau})$. Thus for all $\tau \in[0, \bar{\tau})$ there exists $\omega=\omega(\tau)>0$ such that $F(\omega(\tau), \tau)=0$. Consequently, for all $\tau \in[0, \bar{\tau})$, and from (5.2), there exists a unique $\theta(\tau) \in[0,2 \pi]$, such that

$$
\left\{\begin{array}{l}
\cos (\theta(\tau))=\frac{\left[a_{4}(\tau)-a_{1}(\tau) a_{3}(\tau)\right] \omega^{2}(\tau)-a_{2}(\tau) a_{4}(\tau)}{a_{4}^{2}(\tau)+a_{3}^{2}(\tau) \omega^{2}(\tau)} \\
\sin (\theta(\tau))=\frac{a_{3}(\tau) \omega^{3}(\tau)+\left[a_{1}(\tau) a_{4}(\tau)-a_{2}(\tau) a_{3}(\tau)\right] \omega}{a_{4}^{2}(\tau)+a_{3}^{2}(\tau) \omega^{2}(\tau)} .
\end{array}\right.
$$

Then one can check that $i \omega(\tau)$ is a root of (3.2) if and only if $\tau \omega(\tau)=\theta(\tau)+2 k \pi$, for $k \in \mathbb{N}$, that is if $\tau$ is a root of the function

$$
Z_{k}(\tau)=\tau-\frac{\theta(\tau)+2 k \pi}{\omega(\tau)}, \quad \tau \in[0, \bar{\tau}), k \in \mathbb{N} .
$$

One can easily verify that

$$
Z_{k}(0)<0 \quad \text { for all } k \in \mathbb{N}, \quad \text { and } \quad Z_{k}(\tau)>Z_{k+1}(\tau), \quad \text { for } k \in \mathbb{N} .
$$

Hence, if $Z_{k}$ has no positive root, then no $Z_{j}$ function, with $j>k$, has positive roots. The following theorem deals with the asymptotic stability of the positive steady state.

Theorem 7. Assume (2.8) holds true. If no $\tau \in\left[0, \tau_{\max }\right)$ satisfies (5.3), then the positive steady state $\left(N^{*}, S^{*}\right)$ of system (2.3)-(2.4) is locally asymptotically stable for $\tau \in\left[0, \tau_{\text {max }}\right)$. Assume there exists $0<\bar{\tau}<\tau_{\max }$ such that (5.3) is fulfilled for $\tau \in[0, \bar{\tau})$. The following assertions hold:

(i) If $Z_{0}(\tau)$ has no root on the interval $[0, \bar{\tau})$, then the positive steady state of system (2.3)-(2.4) is locally asymptotically stable for $\tau \in[0, \bar{\tau})$.

(ii) If $Z_{0}(\tau)$ has at least one positive root on the interval $[0, \bar{\tau})$, say $\tau_{c}$, then the positive steady state of system (2.3)-(2.4) is locally asymptotically stable for $\tau \in\left[0, \tau_{c}\right)$, and loses its stability when $\tau=\tau_{c}$. A finite number of stability switches may occur as $\tau$ increases and passes through roots of the $Z_{k}$ functions. Moreover, if

$$
\operatorname{sign}\left\{2 \omega^{2}\left(\tau_{c}\right)+b_{1}\left(\tau_{c}\right)\right\} \operatorname{sign}\left\{\frac{d Z_{0}}{d \tau}\left(\tau_{c}\right)\right\}>0,
$$

then a Hopf bifurcation occurs at $\left(N^{*}, S^{*}\right)$ when $\tau=\tau_{c}$.

Proof. First recall that, from Proposition 5, the positive steady state of system (2.3)-(2.4) is locally asymptotically stable when $\tau=0$. If condition (5.3) is never satisfied on the interval $\left[0, \tau_{\max }\right)$, then (3.2) has no purely imaginary root. Consequently, the stability of $\left(N^{*}, S^{*}\right)$ cannot be modified. The first conclusion of the theorem then follows. 
Assume now (5.3) holds true for $\tau \in[0, \bar{\tau})$. If $Z_{0}$ has no root in $[0, \bar{\tau})$, then, thanks to the remark preceding the theorem (see (5.4)), equation (3.2) has no purely imaginary root for $\tau$ in $[0, \bar{\tau})$ and the conclusion is straightforward.

If $Z_{0}$ has at least one positive root $\tau_{c}$ then, from the definition of $Z_{k}$ functions, (3.2) has purely imaginary roots $\pm i \omega$ when $\tau=\tau_{c}$, with $\omega=\omega\left(\tau_{c}\right)$. The stability of $\left(N^{*}, S^{*}\right)$ switches from stable to unstable as $\tau$ passes through $\tau_{c}$. Other stability switches occur when $\tau$ passes through roots of the $Z_{k}$ functions (see Beretta and Kuang [7]).

Finally, the Hopf bifurcation Theorem says that a Hopf bifurcation occurs at $\left(N^{*}, S^{*}\right)$ when $\tau=\tau_{c}$ if

$$
\left.\frac{d \operatorname{Re}(\lambda(\tau))}{d \tau}\right|_{\tau=\tau_{c}}>0
$$

where $\lambda(\tau)$ is a branch of eigenvalues of (3.2) such that $\lambda\left(\tau_{c}\right)=i \omega\left(\tau_{c}\right)$. From [7], we know that

$$
\operatorname{sign}\left(\left.\frac{d \operatorname{Re}(\lambda(\tau))}{d \tau}\right|_{\tau=\tau_{c}}\right)=\operatorname{sign}\left(\frac{\partial f}{\partial z}\left(\omega^{2}\left(\tau_{c}\right), \tau_{c}\right)\right) \operatorname{sign}\left(\frac{d Z_{0}\left(\tau_{c}\right)}{d \tau}\right) .
$$

Since

$$
\frac{\partial f}{\partial z}(z, \tau)=2 z+b_{1}(\tau)
$$

the last result of the theorem is proven. This ends the proof.

The above theorem stresses the possibility for a stability switch to occur. However, determining analytically the roots of the $Z_{k}$ functions is very difficult - and certainly impossible at this time. Consequently, in the next section, we numerically investigate these stability switches.

\section{Numerical simulations}

Throughout this section, we focus on numerical simulations showing stability switches, as mentioned in Theorem 7. Hence, we concentrate ourselves on the asymptotic behavior of the positive steady state, the behavior of the trivial steady state being easily determined (see Theorem 4). We first choose the introduction rate $\beta$ as a Hill function $[11,16]$, that is a smooth decreasing and positive function, defined by

$$
\beta(S)=\beta_{0} \frac{\theta^{n}}{\theta^{n}+S^{n}}, \quad \beta_{0}, \theta \geq 0, n>1 .
$$

The parameter $\beta_{0}$ represents the maximal rate of introduction in the proliferating phase, $\theta$ is the value for which $\beta$ attains half of its maximum value, and $n$ is the sensitivity of the rate of introduction.

The positive steady state exists if and only if (2.10) is satisfied, that is

$$
\delta<\beta_{0} \quad \text { and } \quad 0 \leq \tau<\tau_{\max }:=\frac{1}{\gamma} \ln \left(\frac{2 \beta_{0}}{\delta+\beta_{0}}\right) .
$$


From Theorem 7, the stability of the positive steady state depends upon the roots of the $Z_{k}$ functions. Consequently, our numerical investigations will consist in determining, for a given set of parameters $\left(\delta, \gamma, \beta_{0}, \theta, n\right)$, the roots of the functions $Z_{0}$ and if necessary $Z_{k}$ for $k \geq 1$. When the $Z_{k}$ functions have positive roots then the positive steady state undergoes a stability switch.

First, from now on and up to the end of this section, we normalize the value of $\theta$ to $\theta=1$. This has no consequence on the quantitative behavior of the system (2.3)-(2.4). Then, the number of parameters determining the stability of the positive steady state equals four: $\delta, \gamma, \beta_{0}$ and $n$. Two parameters, $\delta$ and $\gamma$, are mortality rates, whereas $\beta_{0}$ and $n$ characterize the introduction rate.

For each pair of parameters, we numerically compute the $Z_{k}$ functions, and determine whether they have positive roots or not. One may notice that in some cases, the range of the parameters is limited, mainly because (2.10) must hold for the positive steady state to exist. Two diagrams, which appeared to be the most interesting for the stability switch analysis, are displayed in Figure 1 . They show, in the $(n, \gamma)$ - and $(\delta, \gamma)$-planes, the areas where stability switches can occur.
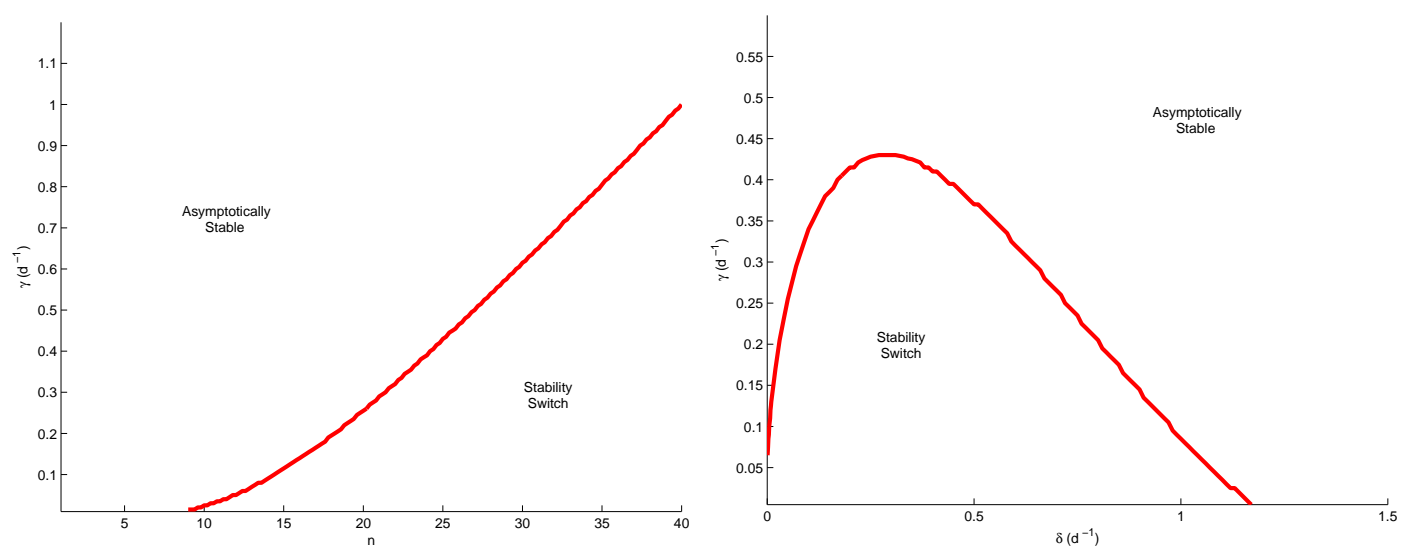

Figure 1: Left: In the $(n, \gamma)$-plane, areas of asymptotic stability and stability switch of the positive steady state. Other parameters are $\beta_{0}=1.77 \mathrm{~d}^{-1}$ and $\delta=0.05 \mathrm{~d}^{-1}$. Right: In the $(\delta, \gamma)$-plane, areas of asymptotic stability and stability switch of the positive steady state. Other parameters are $\beta_{0}=1.77 \mathrm{~d}^{-1}$ and $n=20$.

The first diagram shows that as $n$ increases and reaches large values stability switches are more likely to occur, independently of the value of the apoptosis rate $\gamma$. For small values of $n$ (indeed, $n<9)$, the positive steady state is asymptotically stable for $\tau \in\left[0, \tau_{\max }\right)$. Pujo-Menjouet et al. $[20,21]$ noticed, for the model (2.1)-(2.2) with the introduction rate $\beta$ depending only upon the nonproliferating cell population $N(t)$, that oscillations could be observed for $n \geq 10$, which is similar to what we obtain here. For large values of $n$, the function $\beta$ is similar to a step function (see, for instance, Pujo-Menjouet and Mackey [21]) and then system (2.3)-(2.4) can possibly be studied in a different way. Moreover, it appears that for any value of $\gamma$ it is possible to find a value of $n$ for which a stability switch can occur, and then the positive steady state can be destabilized.

The second diagram shows very different conclusions. Stability switch can occur for smaller values of $\gamma$, and for small values of $\delta$. For instance, $\delta$ cannot be larger than $\beta_{0}$ for the positive 
steady state to be well defined, and $\beta_{0}=1.77 \mathrm{~d}^{-1}$ in this case, which is much larger than the maximum value of $\delta$ for which a stability switch can occur. The largest area is obtained for $\delta$ about $0.3-0.4 \mathrm{~d}^{-1}$, and $\gamma$ ranges then in $\left[0,0.43 \mathrm{~d}^{-1}\right]$.

Diagrams in Figure 1 stress out the complexity of the positive steady state behavior, a stability switch being hard to determine. They also show that for realistic values of the mortality rates (that is $\gamma<0.5 \mathrm{~d}^{-1}$ and $\delta<0.5 \mathrm{~d}^{-1}$, see Mackey [16]), stability switches are likely to occur. Fixing some of the parameters, we now focus on the values of the delay $\tau$ corresponding to stability switches.

Let $\delta=0.05 \mathrm{~d}^{-1}, \beta_{0}=1.77 \mathrm{~d}^{-1}$ and $n=20$. Then according to Figure 1 , for $\gamma \leq 0.26 \mathrm{~d}^{-1}$ stability switches occur. Critical values of $\tau$ for which stability changes are represented in Figure 2. It is noticeable that stability switches are, in the current case, associated only with roots of $Z_{0}(\tau)$, the $Z_{k}$ functions, with $k \geq 1$, being always strictly negative with this choice of parameters. Hence, when stability switches occur, one always observes a destabilization of the positive steady state followed by a re-stabilization.
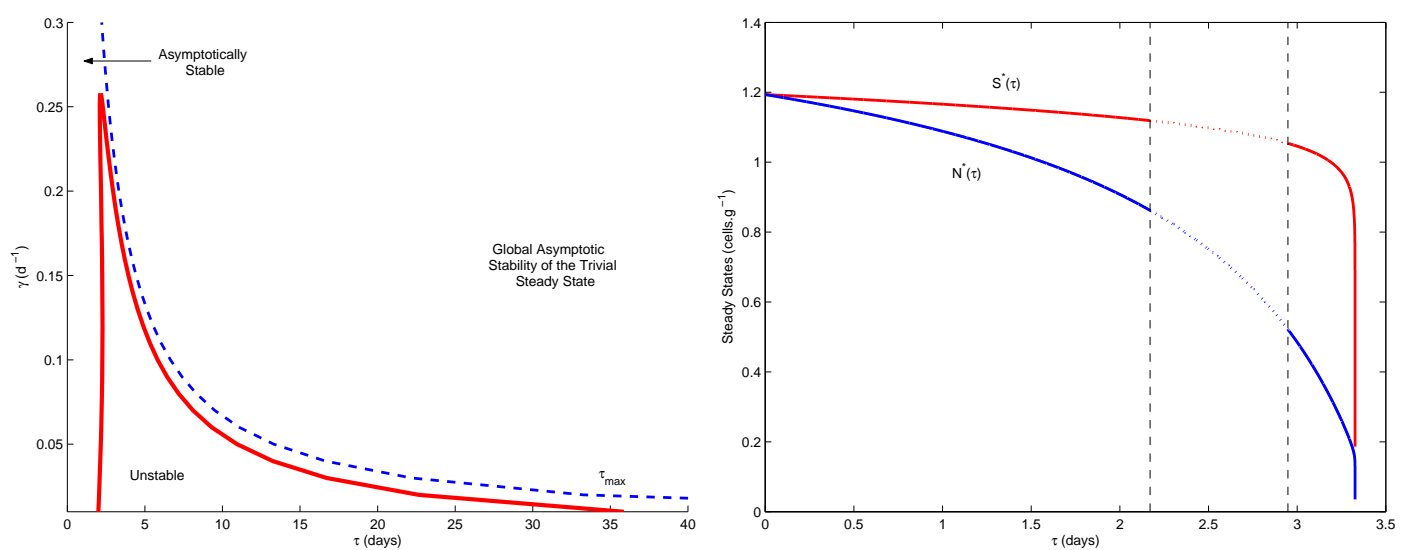

Figure 2: Left: Critical values of $\tau$ for which a stability switch occurs (solid line). The apoptosis rate $\gamma$ ranges in $\left[0,0.26 \mathrm{~d}^{-1}\right]$, and for a given value of $\gamma$ the delay $\tau$ cannot be larger than $\tau_{\max }$ (dashed line). For each value of $\gamma$ two critical values of $\tau$ correspond to stability switch. Right: The positive steady state values, $S^{*}(\tau)$ (top) and $N^{*}(\tau)$ (bottom). The apoptosis rate is equal to $0.2 \mathrm{~d}^{-1}$, so as indicated by the picture on the left-hand side, the steady state is stable (solid lines) for $\tau<2.17$ days and $\tau>2.95$ days, and unstable otherwise (dashed lines). When $\tau$ reaches $\tau_{\max } \approx 3.33$ days, the positive steady state approaches the trivial steady state.

From Figure 2, one notices that for each value of $\gamma$ the positive steady state undergoes a bifurcation as $\tau$ increases, then the steady state stabilizes anew just before it disappears (and the trivial steady state becomes the only steady state). The shape of the unstable zone in Figure 2 is not trivial, and appears very difficult to determine analytically. The numerical investigation performed in this section seems, at this time, the only way to obtain information on the dependence of the positive steady state stability on the parameters.

Steady state values, as functions of $\tau$, are presented in Figure 2, for the above mentioned values 
of the parameters and $\gamma=0.2 \mathrm{~d}^{-1}$. Stability occurs when $\tau<2.17$ days, then the steady state becomes unstable (and one can check that a Hopf bifurcation occurs when $\tau=2.17$ days), then stability switches and the steady state is stable again. Unstable solutions of (2.3)-(2.4) are obtained in this case, using the MATLAB solver dde23 [22], and displayed in Figure 3. They correspond to solutions obtained for $\delta=0.05 \mathrm{~d}^{-1}, \gamma=0.2 \mathrm{~d}^{-1}, \beta_{0}=1.77 \mathrm{~d}^{-1}, n=20$ (that is the situation in Figure 2, right), and $\tau=2.5$ days. Unstable solutions periodically oscillate, with a period about 11 days. One may note that amplitudes of the oscillations are larger for the nonproliferating cell population than for the total population. Moreover, initial conditions are constant on the interval $[-\tau, 0]$, with values such that (2.5) holds true. Consequently, $S(t) \geq N(t)$ for all $t \geq 0$.
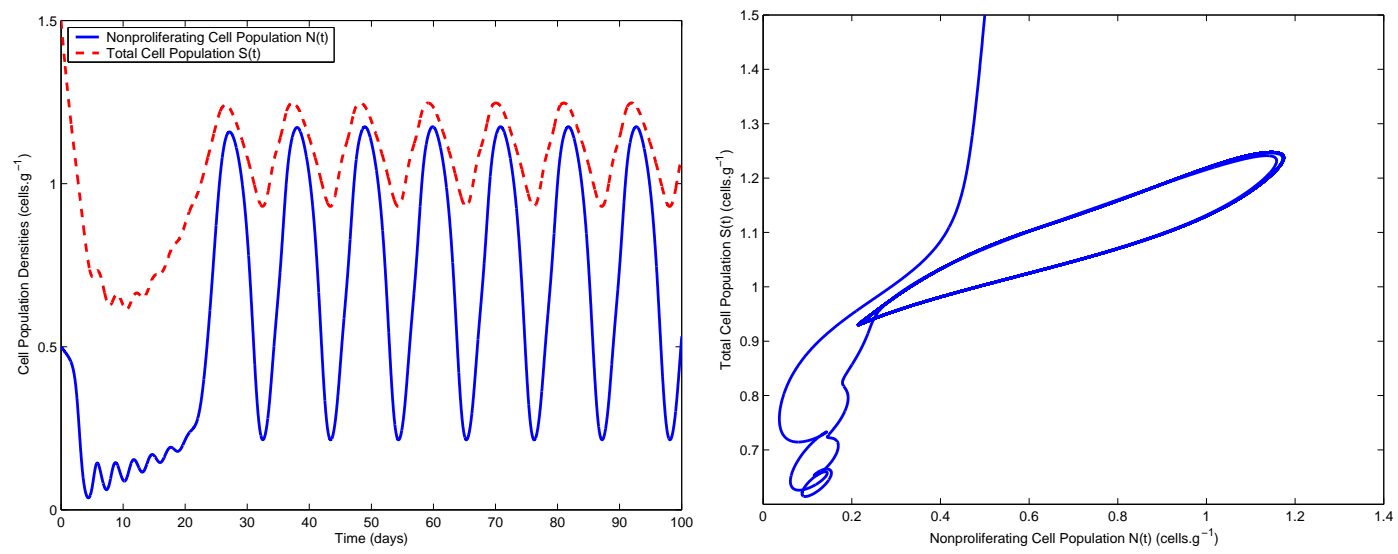

Figure 3: Unstable solutions of system (2.3)-(2.4), $S(t)$ (dashed line) and $N(t)$ (solid line). Parameters are $\delta=0.05 \mathrm{~d}^{-1}, \gamma=0.2 \mathrm{~d}^{-1}, \beta_{0}=1.77 \mathrm{~d}^{-1}, n=20$ and $\tau=2.5$ days. Left: Periodic oscillations, period about 11 days. Right: Limit cycle in the $(N, S)$-plane.

\section{Discussion}

This paper is the follow-up of a previous publication [11]. We considered a nonlinear model of hematopoietic stem cell dynamics, first introduced by Mackey [16], in which the whole cell population triggers introduction of nonproliferating cells in the proliferating phase. In [11], a particular case was analyzed, when the mortality rates of the proliferating and the nonproliferating cells were supposed to be the same. We no longer considered this assumption and performed a complete analysis of the resulting system. In particular, we obtained necessary and sufficient conditions for the global asymptotic stability of the trivial steady state. We also investigated the stability of the unique positive steady state by applying a method developed by Beretta and Kuang [7] for characteristic equations with delay-dependent coefficients. This allowed to state the existence of stability switches for the positive steady state, the analytical proof of such stability switches remaining very hard to complete. A numerical investigation proved efficient to determine stability switches and 
their consequences on the cell population dynamics.

The simulations performed in Section 6 showed, first, that the stability of the positive steady state strongly depends on the parameters. Only two stability diagrams have been shown (Figure 1), yet each parameter contributes to the complexity of the steady state behavior (not shown here). Often, as shown in Figure 1, the influence of a parameter on the stability is very difficult to establish and cannot be easily predicted, or proven analytically. The similarity between the results obtained above and those related to Mackey's model [21] probably indicates that the nature of the trigger signal for introduction in the proliferating phase is still not sure at this time. Second, the numerical results showed that the positive steady state can easily become unstable, by varying one parameter. The instability seems to be characterized by oscillating solutions. This is a well-known feature of hematopoietic stem cell dynamics model, which has been noted several times (see, for instance, Mackey [16], Pujo-Menjouet and Mackey [21], Adimy et al. [4], Crauste [11], and the references therein). Oscillations in cell numbers can be characteristic of blood diseases [12, 14, 20]. Amplitudes and period of the oscillations can allow to distinguish between hematopoietic diseases. These latter depend on the parameters of the system [20] and the time delay $\tau$ [4].

Time delays are known to modify the stability of systems that would not exhibit stability switch in the absence of delay. In various studies of biological models, delays happened to be responsible for complex behaviors. Delays have been stressed to be at the root of instabilities in models of hematopoietic stem cell dynamics since the first model on this topic by Mackey [16]. Some parameters (for instance, the apoptosis rate $\gamma$ ) have been identified as key parameters for the appearance of a blood disease [8], however this could not be without considering the delay in these models. The time delay $\tau$ describes an average cell cycle duration and consequently has a strong biological meaning, which can be related to experimental measurements. Not only the delay has been shown to induce instability, but also, as detailed in Section 6, to stabilize an unstable system (through a stability switch). This important role of the delay can be investigated to determine experimental protocols aiming at eliminating or controlling some diseases (see Colijn et al. [10]).

\section{References}

[1] M. Adimy, F. Crauste. Global stability of a partial differential equation with distributed delay due to cellular replication. Nonlinear Analysis, 54 (2003), 1469-1491.

[2] M. Adimy, F. Crauste. Modelling and asymptotic stability of a growth factor-dependent stem cells dynamics model with distributed delay. Discret. Cont. Dyn. Sys. Ser. B, 8 (2007), No. 1, 19-38.

[3] M. Adimy, F. Crauste, L. Pujo-Menjouet. On the stability of a maturity structured model of cellular proliferation. Discret. Cont. Dyn. Sys. Ser. A, 12 (2005), No. 3, 501-522.

[4] M. Adimy, F. Crauste, S. Ruan. Stability and Hopf bifurcation in a mathematical model of pluripotent stem cell dynamics. Nonlinear Analysis: Real World Applications, 6 (2005), No. 4, 651-670. 
[5] M. Adimy, F. Crauste, S. Ruan. Periodic Oscillations in Leukopoiesis Models with Two Delays. J. Theo. Biol., 242 (2006), 288-299.

[6] J. Bélair, M.C. Mackey, J.M. Mahaffy. Age-structured and two-delay models for erythropoiesis. Math. Biosci., 128 (1995), 317-346.

[7] E. Beretta, Y. Kuang. Geometric stability switch criteria in delay differential systems with delay dependent parameters. SIAM J. Math. Anal., 33 (2002), No. 5, 1144-1165.

[8] S. Bernard, J. Bélair, M.C. Mackey. Oscillations in cyclical neutropenia: New evidence based on mathematical modeling. J. Theor. Biol., 223 (2003), 283-298.

[9] F.J. Burns, I.F. Tannock. On the existence of a $G_{0}$ phase in the cell cycle. Cell Tissue Kinet., 19 (1970), 321-334.

[10] C. Colijn, C. Foley, M.C. Mackey. G-CSF treatment of canine cyclical neutropenia: A comprehensive mathematical model. Exper. Hematol., 35 (2007), No. 6, 898-907.

[11] F. Crauste. Global Asymptotic Stability and Hopf Bifurcation for a Blood Cell Production Model. Math. Bio. Eng., 3 (2006), No. 2, 325-346.

[12] P. Fortin, M.C. Mackey. Periodic chronic myelogenous leukemia: Spectral analysis of blood cell counts and etiological implications. Brit. J. Haematol., 104 (1999), 336-345.

[13] J. Hale, S.M. Verduyn Lunel. Introduction to functional differential equations. Applied Mathematical Sciences 99. Springer-Verlag, New York, 1993.

[14] C. Haurie, D.C. Dale, M.C. Mackey. Cyclical neutropenia and other hematological disorders: A review of mechanisms and mathematical models. Blood, 92 (1998), No. 8, 2629-2640.

[15] L.G. Lajtha. On DNA labeling in the study of the dynamics of bone marrow cell populations, in: Stohlman, Jr., F. (Ed), The Kinetics of Cellular Proliferation, Grune and Stratton, New York (1959) 173-182.

[16] M.C. Mackey. Unified hypothesis of the origin of aplastic anaemia and periodic hematopoiesis. Blood, 51 (1978), 941-956.

[17] M.C. Mackey, R. Rudnicki. Global stability in a delayed partial differential equation describing cellular replication. J. Math. Biol., 33 (1994), 89-109.

[18] M.C. Mackey, R. Rudnicki. A new criterion for the global stability of simultaneous cell replication and maturation processes. J. Math. Biol., 38 (1999), 195-219.

[19] J.M. Mahaffy, J. Bélair, M.C. Mackey. Hematopoietic model with moving boundary condition and state dependent delay. J. Theor. Biol., 190 (1998), 135-146.

[20] L. Pujo-Menjouet, S. Bernard, M.C. Mackey. Long period oscillations in a $G_{0}$ model of hematopoietic stem cells. SIAM J. Appl. Dyn. Systems, 4 (2005), No. 2, 312-332. 
[21] L. Pujo-Menjouet, M.C. Mackey. Contribution to the study of periodic chronic myelogenous leukemia. C. R. Biologies, 327 (2004), 235-244.

[22] L.F. Shampine, S. Thompson. Solving DDEs in MATLAB. Appl. Numer. Math., 37 (2001), $441-458$. 